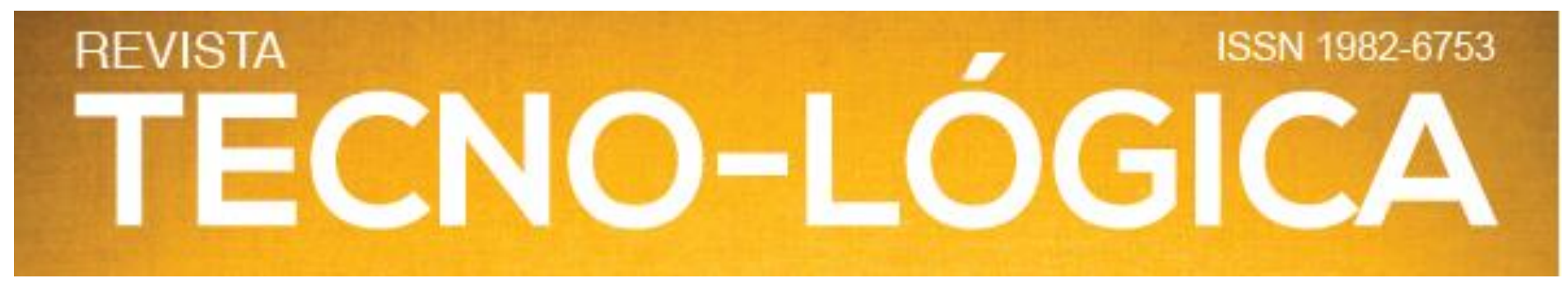

\title{
BARRAS DE FIBRA DE VIDRO, UMA ALTERNATIVA INOVADORA E SUAS POTENCIALIDADES: REVISÃO BIBLIOGRÁFICA
}

\author{
Adriano Ineia*, William de Oliveira Pol, Julia Catiane Arenhardt Braun, Luizmar da Silva Lopes Junior \\ Programa de Pós-Graduação em Engenharia Civil e Ambiental, Faculdade de Engenharia e Arquitetura - FEAR, Universidade de \\ Passo Fundo, Av. Brasil Leste, 285, Bairro São José, 99052-900, Passo Fundo, Brasil.
}

*E-mail: adri.ano.ineia@hotmail.com

Recebido em: 05/02/2021

Aceito em: 30/06/2021

DOI: 10.17058/tecnolog.v25i2.16214

\section{RESUMO}

O desempenho, eficiência, resiliência e sustentabilidade de uma estrutura são parâmetros fundamentais para promover a qualidade de vida e desenvolvimento econômico de uma infraestrutura. Atualmente, as patologias como corrosão são problemas sérios que oneram os cofres públicos e o consumidor final, além de gerar maiores importunos ao longo do tempo. Por isso, o emprego de materiais alternativos e de alto desempenho ganham cada vez mais atenção, devido à alta complexidade de gerenciamento, manutenção e adaptação resiliente às mudanças sociais, climáticas, poluição acentuada, diminuição dos recursos financeiros e entre outras durante todo o seu ciclo de vida da infraestrutura. Portanto, as barras de GFRP (Glass-Fiber-Reinforced Polymer) ganham evidência por serem inertes a corrosão, sendo empregada como uma ferramenta de reparo, manutenção e emprego em novas obras civis. Esse material associado a tecnologia, possibilita a redução de custo e melhoria no comportamento da estrutura. O plano de fundo deste artigo é de fomentar estudos futuros, vislumbrando desenvolver uma norma brasileira de dimensionamento de concreto armado (CA) GFRP. O domínio deste assunto aos profissionais, gestores e envolvidos na concepção e implementação de sistemas de infraestrutura e obras civis é fundamental, pois permite um planejamento sistêmico, otimizado e coerente as necessidades, minimizando custos, mas sem comprometer a qualidade da estrutura.

Palavras-chave: Barras de reforço de GFRP. Concreto reforçado com fibra. Polímero reforçado com fibra de vidro GFRP.

\section{Introdução}

O compósito de fibras de vidro em barras, advém da premissa de contornar os impactos ambientais e as características físicas, químicas e as limitações de aço. As denominadas barras GFRP (Glass-Fiber-Reinforced Polymer), são produzidas com materiais compósitos reforçados com fibras de vidro. No mundo, apesar de ser algo novo e desconhecido no mercado da construção civil brasileira, esta tecnologia possui algumas normas, como a japonesa, americana e canadense.

A estrutura deste material se apresenta promissora por ser resistente aos efeitos deletérios como o ataque de álcalis do cimento. A tecnologia que está por trás das barras GFRP, deriva da matriz inteligente e resina polimérica, ou seja, dependendo a formulação a barra demonstra resistência ao fogo, ataque químico, salinidade, umidade e degelo. Esse material da construção civil confere durabilidade, alta sustentabilidade e resistência a fadiga [1].

A barra GFRP é um material anisotrópico, tendo a sua maior resistência no sentido eixo longitudinal. As características e propriedades mecânicas deste material dependem de inúmeros fatores como volume e tipo da fibra e resina, orientação (sentido), controle de qualidade na fabricação e execução na obra $[2,3]$.

As propriedades das barras de polímeros reforçados com fibra de vidro (GFRP), permitem que ele seja empregado em reparos, reabilitação e novas obras de infraestrutura, principalmente em condições adversas. Pois, elas conferem à estrutura uma durabilidade prolongada de sua integridade. 


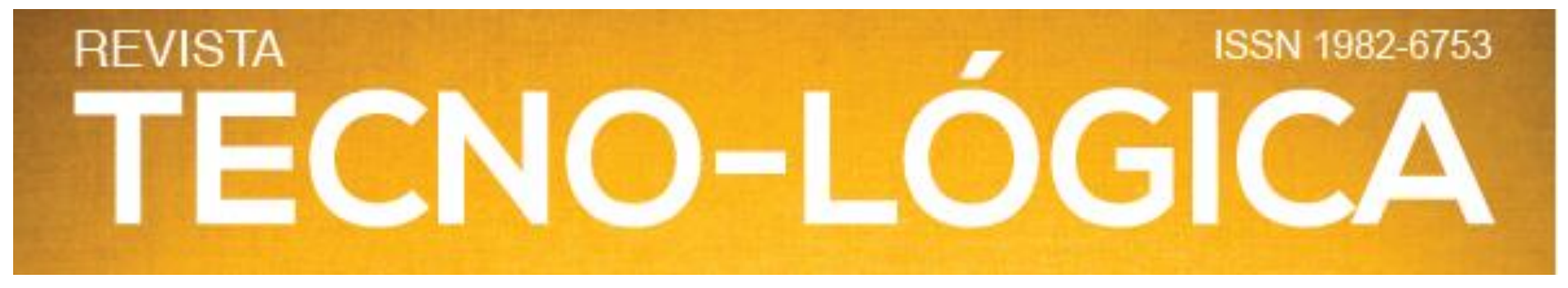

Entretanto, o material apresenta uma ampla variação das propriedades físico-mecânicas do GFRP [4].

O mecanismo de aderência entre o concreto e as barras de reforçados com fibra de vidro é diferente do com aço convencional, devido à diferença dos materiais, dentre elas o módulo da elasticidade, expansão térmica, propriedades mecânicas e entre outros. Este comportamento de ligação está intrínseco a inúmeros fatores como diâmetro da barra, cobrimento, comprimento de encaixe, superfície da barra e correlatos [2].

Mesmo sendo um material promissor, ainda não se esgotaram estudos diretos e correlatos as propriedades, comportamento e desempenho deste material. Portanto, o plano de estudo deste artigo, tem por ambição subsidiar novos estudos nesta área e fomentar num futuro uma norma brasileira de dimensionamento. A estratégia do estudo consiste em uma pesquisa bibliográfica com ênfase em barras de polímeros reforçados com fibra de vidro, visando introduzir uma discussão detalhada das propriedades, do comportamento estrutural e desempenho do GFRP.

\section{(GFRP) \\ 2 Barras de fibra de vidro impregnada de polímero}

Alguns conceitos e termos considerados relevantes a barra de fibra de vidro (GFRP), foram especificados e abordados como forma de fundamentar, justificar e legitimar o respectivo estudo e nortear o leitor com uma contextualização que facilite seu entendimento. mundial

\subsection{Considerações iniciais do GFRP sob perspectiva}

O estopim da utilização de técnicas que empregam compósitos na construção civil, advém do governo japonês. Por se haver a necessidade de recuperar as estruturas danificadas, mas também com o intuito de conceder a estrutura resiliente e preventiva em situações adversas como abalos sísmicos. O GFRP surgiu no Japão como necessidade, entretanto se mostrou uma técnica resiliente e duradoura, assim não demorou muito para se proliferar pelo mundo [2].

$\mathrm{Na}$ conjuntura mundial atual, as barras de fibra de vidro estão bastante difundidas. O primeiro país a normatizar uma norma de dimensionamento de estruturas com GFRP, foi o Canadá por meio da CSA S806. A norma fornece fatores parciais de resistência, não levando em consideração a redução de resistência, outra particularidade é que o projetista não leva em consideração a ruptura, mas sim o esmagamento do concreto [5].

$\mathrm{Na}$ Europa, como exemplo a Grã-Bretanha, a norma legisladora é a ISTRUDTE [42]. Ela deriva da norma britânica BS8110 [6], tendo alterações de melhorias no dimensionamento.
De modo geral, a União Europeia adotou a norma britânica modificada como norma padrão no dimensionamento de estruturas de concreto armado com GFRP, com o adendo do Eurocode. Dentre as recomendações, se encontram um conjunto de fatores de segurança parciais para resistência, que consideram o comportamento estrutural de curto e longo prazo, entretanto uma mazela é a indicação clara de falhas devido a aplicação de fatores de reduções parciais [5].

Migrando para o oriente, temos a norma japonesa JSCE (1997), ela se origina da norma de concreto armado em aço JSCE 1986, tendo como recomendação de dimensionamento o estado limite de serviço e estado limite último. A norma egípcia teve a formalização do dimensionamento de estruturas de concreto armado com GFRP, pela norma Egyptian Ministry of Housing, ela é uma ramificação da norma de concreto armado com aço [2, 7 , 8].

\subsection{Vantagens do GFRP}

Dentre as vantagens desse tipo de material se destacam a leveza, resistência, instalação fácil e rápida, maior durabilidade em ambientes agressivos, bom isolamento térmico e acústico e transparência eletromagnética [9]. Sendo um material leve, ele possui maior facilidade de manejo e transporte, em comparação com materiais convencionais e mais pesados, como o aço. A transparência eletromagnética permite que não ocorra a interferência de ondas de rádio em construções como edifícios, sem prejudicar a resistência e estética da obra [10].

A sua baixa suscetibilidade à efeitos corrosivos permite que estas estruturas sejam utilizadas em ambientes de condições adversas, em que materiais convencionais sofrem maior efeito negativo. Dentre esses ambientes se destacam ambientes salinos e alcalinos, além de ambientes contendo águas residuárias [11].

Os GFRP são materiais de fácil aplicação e manejo, sendo necessário pouca manutenção durante o tempo de vida útil, além de possuírem a sua principal característica que é a durabilidade, sendo capazes de se adaptar a fatores ambientais, como exposição à radiação ultravioleta, além de ser menos propensos de falhas por fadiga [2]. São materiais que podem ser expostos a ambientes agressivos e são isolantes térmicos, sendo que em situações de incêndio ajudam a evitar propagação de calor e são mais dificilmente afetados pelas chamas. Porém essa é uma área onde ainda faltam estudos do comportamento desses materiais quando expostos ao fogo e à altas temperaturas, sendo um empecilho para a utilização de edifícios [12]. Outras vantagens que podem ser citadas são o seu pequeno peso específico, alta resistência à tração, não condutibilidade elétrica, térmica e magnética [13]. 


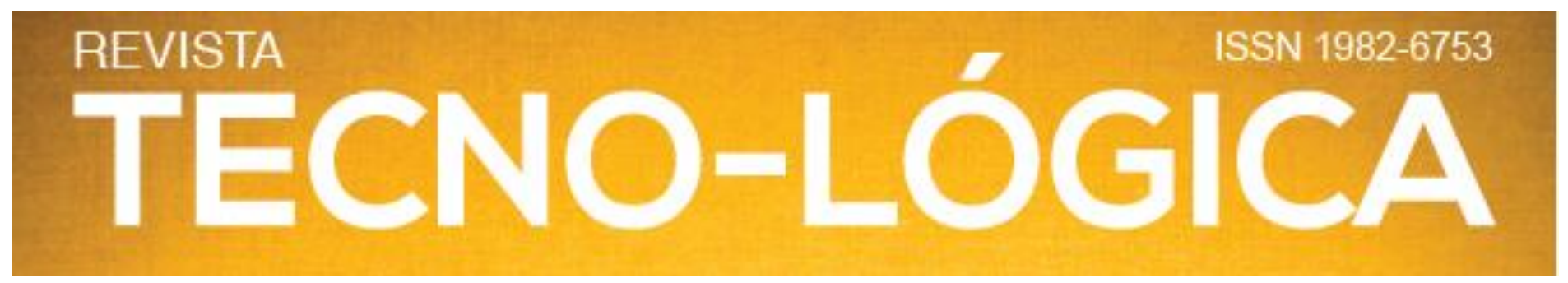

\subsection{Aplicação dos compósitos GFRP}

Devido às características do GFPR, esse material pode ser utilizado em diversas obras de engenharia para otimização das infraestruturas, tanto na fase de construção como restauração. Podem ser utilizados na construção de estruturas, como pontes, coberturas e edifícios, pelas suas propriedades de leveza e durabilidade [10].

O investimento em estudos sobre compósitos, ganha cada vez mais investimento, devido ao comportamento resiliente do material. A aplicação das barras GFRP como armadura cresceu muito nos últimos anos, devido suas propriedades. Um exemplo, na Figura 1 demonstra que o Innovative Bridge Research \& Construction Program (IBRC), empregou a armadura de GFRP nos EUA entre os anos de 1998 a 2001, fazendo o uso dessa técnica em 84 de 175 pontes, superando os 17 projetos executados com aço de alto desempenho e as 30 pontes de concreto de alta resistência [2].

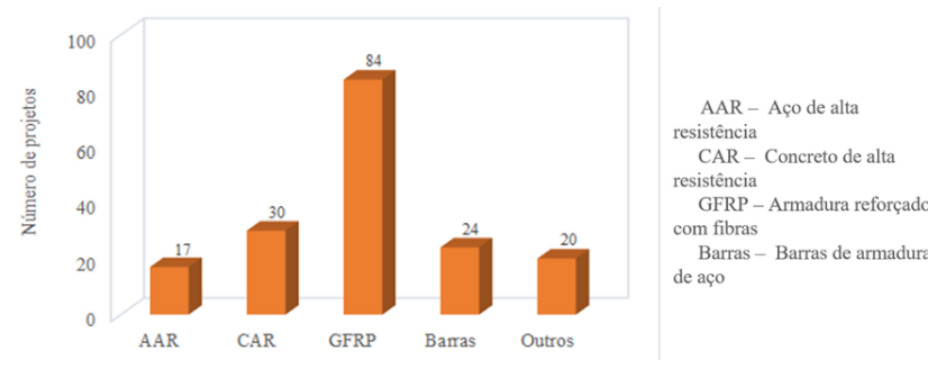

Figure 1: Comparativo de projetos e soluções estruturais de pontes realizados pelo Innovative Bridge Research \& Construction Program (IBRC). Fonte: Adaptado de Ribeiro [2].

Essa técnica construtiva, foi executada no tabuleiro das pontes, reforços de estruturas e armadura. Isso demonstra a versatilidade do FRP, e sua atuação no parâmetro da resiliência da estrutura. Na Figura 2, temos as porcentagens de aplicações do material na execução das pontes.

O material também pode ser utilizado em passarelas de pedestre, sendo que é um material adequado, seguro e apresenta boas respostas quanto às vibrações e conforto dos transeuntes [14].

Como possui características de resistência à corrosão e ambientes quimicamente adversos, esse material pode ser utilizado em estações de tratamento de águas residuárias e torres de arrefecimento proporcionando maior durabilidade à essas estruturas [10].

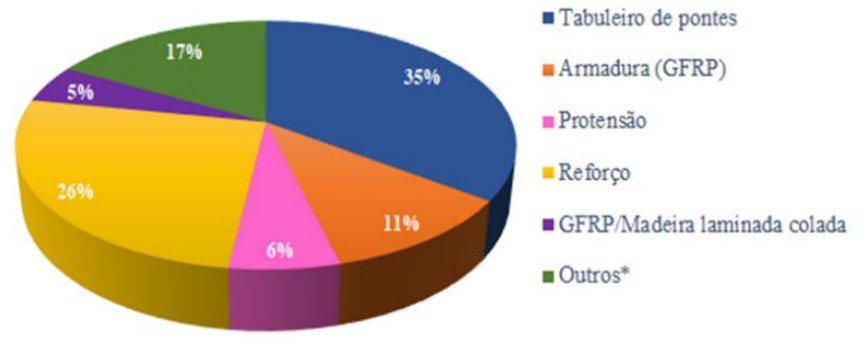

Figura 2: Distribuição das aplicações nas pontes do FRP. Fonte: Adaptado de Ribeiro [2].

Em ambientes de águas salinas é um material que também sofre menos efeitos adversos, assim ele pode ser utilizado em construções marítimas que sofrem os efeitos dos íons cloreto. Os reforços de estruturas convencionalmente utilizados são de aço carbono que acabam sofrendo os efeitos corrosivos nessas condições. Em vista disso, os reforços de GFPR podem ser alternativas de substituição desses materiais. Além disso, a incorporação desses materiais em concretos fabricados a partir de areia de águas marítimas pode amenizar o problema da larga utilização de areia de água doce, que pode ter sua disponibilidade reduzida ao longo do tempo e que é mais escassa em áreas costeiras [15].

Quanto à reabilitação de estruturas, esse material se apresenta como promissor na área. O material pode ser usado na substituição parcial ou total de estruturas como a madeira degradadas, onde a preservação pode ser realizada pelo GFRP. Assim, a restauração de edifícios antigos, como castelos, pode ser realizada evitando o desgaste natural dessas estruturas e ainda preservar seu valor patrimonial [10].

\subsection{Compósitos de GFRP}

As barras de fibra de vidro GFRP, são potenciais alternativas de armadura no concreto armado convencional. Estas barras possuem propriedades anisotrópicas, ou seja, sua maior resistência se encontra no sentido longitudinal. Sua composição consiste em um núcleo de fibras (sintéticas ou minerais) de elevada resistência e impregnadas de matriz polimérica (resina) [13].

O tipo de fibra empregada no núcleo interfere nas propriedades das barras. Na construção civil, são usadas no processo de fabricação das barras as fibras de carbono, aramida e fibra de vidro (motivo de estudo). No quesito financeiro, a fibra de vidro apresenta o melhor custo-benefício. Atualmente, as fibras de vidro comercialmente disponíveis para construção civil são: vidroE, Vidro-S e Vidro álcali resistente [13, 16].

As GFRP costumeiramente, são fabricadas pelo processo de pultrusão, ao final apresentam características visuais similares 


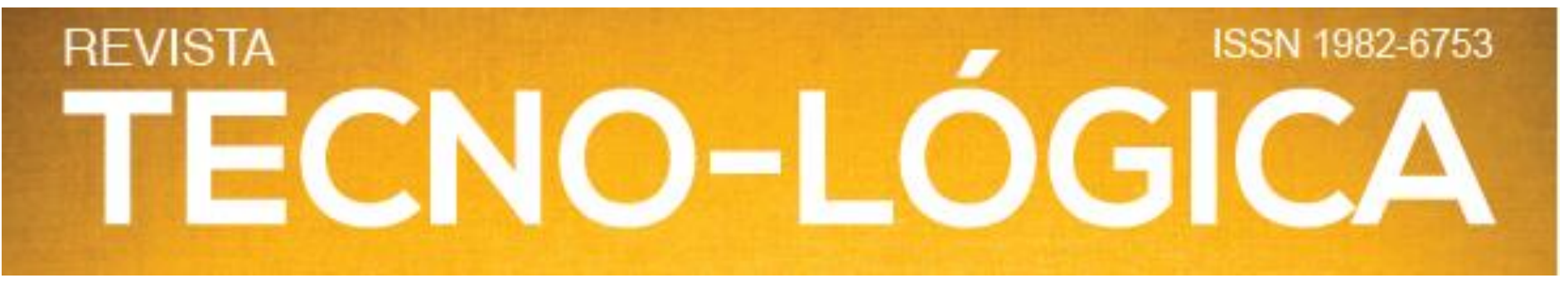

a barras de aço, entretanto propriedades mecânicas diferentes. Uma particularidade destas barras é a dobra das barras, elas devem ser manufaturadas de forma pré-definida, conforme previsto em projeto, pois não se consegue dobrar em canteiro de obra.

\section{GFRP}

\subsection{Matriz polimérica e caracterização das barras de}

Tabela 1: Propriedades das matrizes poliméricas dos compósitos de FRP.

\begin{tabular}{|c|c|c|c|}
\hline \multirow{2}{*}{ PROPRIEDADES } & \multicolumn{3}{|c|}{ MATRIZ } \\
\hline & Poliéster & Epóxi & Vinil Éster \\
\hline Massa específica $(\mathrm{g} / \mathrm{cm} 3)$ & $1,20-1,40$ & $1,20-1,40$ & $1,15-1,35$ \\
\hline Resistência à tração (MPa) & $34,5-104$ & $55-130$ & $73-81$ \\
\hline Módulo Longitudinal (GPa) & $2,1-3,45$ & $2,75-4,10$ & $3,0-3,5$ \\
\hline Coeficiente de Poisson & $0,35-0,39$ & $0,38-0,40$ & $0,36-0,39$ \\
\hline $\begin{array}{l}\text { Coeficiente de dilatação térmica (10- } \\
\left.\qquad 6 /{ }^{\circ} \mathrm{C}\right)\end{array}$ & $55-100$ & $45-65$ & $50-75$ \\
\hline Teor de umidade $(\%)$ & $0,15-0,60$ & $0,08-0,15$ & $0,24-0,30$ \\
\hline
\end{tabular}

Fonte: Federation International Du Beton [18] e Couto [13].

A priori os compósitos de FRP, apresentam um peso específico baixo, na prática de 4 a 6 vezes mais leve que o aço. Isso otimiza o processo de logística, manuseio e instalação da estrutura. Tornando a obra mais rentável por minorar gastos com instalações $[2,19]$.

O coeficiente de dilatação térmica das barras de GFRP, está intrinsecamente ligado à direção e composição. Os principais parâmetros que podem variar esse comportamento da barra são a direção longitudinal à direção transversal, a tipologia da fibra, a dosagem e proporção de resina em comparação a volumetria de fibras. Na literatura, se encontra uma relação volumétrica de fibras variando de 05 a $0,7 \times 10-6{ }^{\circ} \mathrm{C}$, conforme citado no comparativo da Tabela 1 [2].

A aderência das barras de GFRP, está ligado ao processo industrial fabril, pois é ele que confere as propriedades mecânicas e superfície da barra. Uma particularidade desta barra, é de que a resistência de aderência se dá resina para as fibras. O processo de fabricação deve ser minucioso, pois existe possibilidade de falha na resina.

\subsection{Processo de fabricação das barras de GFRP}

A matriz polimérica das barras de GFRP, é a responsável por descarregar as tensões entre as fibras que constituem as barras e o concreto adjacente. As resinas empregadas na fabricação das barras são denominadas como termoestáticas (epóxi, vinil éster e poliéster), as propriedades destas se encontram especificadas na Tabela 1 [13]. 


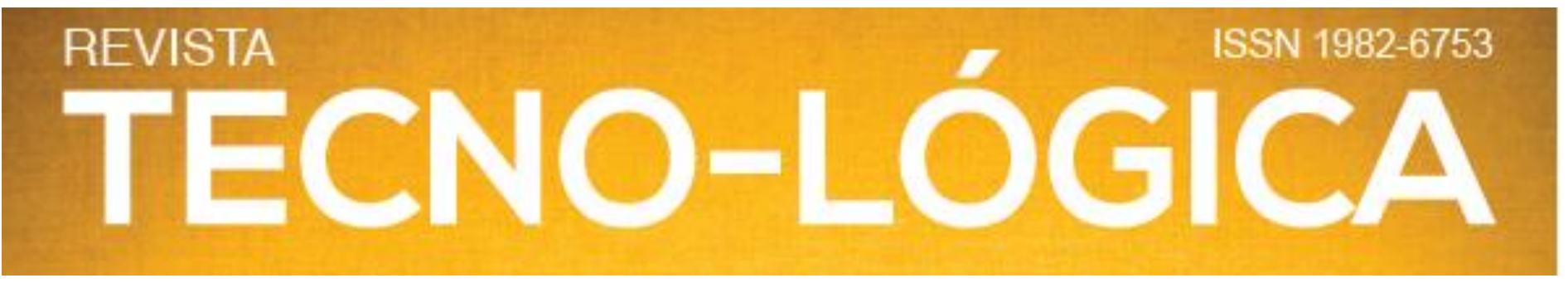

como, tipo de resina, efeito dimensionais e controle de qualidade possuem papel importante nas propriedades mecânicas que podem variar em função destes. Algumas de suas propriedades mecânicas são discutidas a seguir.

\subsection{Comportamento Anisotrópico}

As barras GFRP são de natureza anisotrópicas, possuindo elevada resistência à tração na orientação longitudinal as fibras. Contudo, tal comportamento anisotrópico afeta a resistência ao cisalhamento assim como a aderência junto ao concreto.

\subsection{Densidade}

As barras de GFRP, assim como barras de polímeros reforçados com fibras em geral, possuem uma densidade que variam de $1,25 \mathrm{~g} / \mathrm{cm}^{3}$ a $2,10 \mathrm{~g} / \mathrm{cm}^{3}$ o que em relação ao aço mostrase de 4 a 6 vezes mais leves (Tabela 02). Tal diferença lhe confere menor custo de transporte e facilidade de manuseio, reduzindo o tempo empregado em sua instalação mostrando-se ser uma solução interessante a ser considerada junto a avaliação de viabilidade para armaduras estruturais [19].

\begin{tabular}{cccc}
\hline Aço $\left[\mathrm{g} / \mathrm{cm}^{3}\right]$ & GFRP $\left[\mathrm{g} / \mathrm{cm}^{3}\right]$ & CFRP $\left[\mathrm{g} / \mathrm{cm}^{3}\right]$ & AFRP $\left[\mathrm{g} / \mathrm{cm}^{3}\right]$ \\
\hline 7,90 & 1,25 a 2,10 & 1,50 a 1,60 & 1,25 a 1,40 \\
\hline
\end{tabular}

Fonte: Adaptado de ACI Committee 440 [28].

\subsection{Expansão térmica}

O coeficiente de expansão térmica de uma barra FRP variam nas direções longitudinal e transversal, sendo estres dependentes do tipo de fibra, resina e fração volumétrica da fibra. O coeficiente de expansão térmica longitudinal é regido pelas características das fibras empregadas, enquanto o coeficiente transversal é regido pelas características da resina [22].

Os polímeros reforçados com fibras possuem uma expansão térmica na direção transversal de maior magnitude em relação a direção longitudinal e maior também que a expansão térmica do concrento endurecido [23]. Tal diferença entre os coeficientes transversais de expansão térmica de barras FRP's e do concreto pode causar rachaduras no interior do concreto sob aumento expressivo de temperatura e por conseguinte falha na cobertura do concreto se a ação restritiva do concreto for insuficiente [24-26].

Resultados experimentais mostram que o coeficiente transversal de expansão térmica de barras com fibra de vidro (GFRP) é de $22 \times 10^{-6}{ }^{\circ} \mathrm{C}$ em média, sendo que a razão entre os coeficientes transversal e longitudinal ficam na ordem de 4 . O estudo também mostrara que mantendo uma relação entre a cobertura de concreto e o diâmetro da barra superior a 1,60 é suficiente para evitar rachaduras no concreto sob altas temperaturas até $80^{\circ} \mathrm{C}$, sendo que não há reduções significativas na resistência de união para temperaturas até $60^{\circ} \mathrm{C}$ [23, 26-27]. A Tabela 03 apresenta os coeficientes de expansão térmica nas direções transversal e longitudinal para aço, concreto e barras FRP.

Tabela 3: Coeficientes de expansão térmica nas direções transversal e longitudinal. COEFICIENTES $\left[\mathrm{X} 10^{-6} /{ }^{\circ} \mathrm{C}\right]$

\begin{tabular}{lccccc} 
DIREÇÃO & Concreto & Aço & GFRP & CFRP & AFRP \\
\cline { 2 - 6 } & $7,20 \mathrm{a}$ & \multirow{2}{*}{11,70} & $6,00 \mathrm{a}$ & $-9,00 \mathrm{a}$ & $-6,00 \mathrm{a}$ \\
Longitudinal & 10,80 & & 10,00 & 0,00 & $-2,00$ \\
{$\left[\alpha_{\mathrm{L}}\right]$} & $7,20 \mathrm{a}$ & \multirow{2}{*}{11,70} & $21,00 \mathrm{a}$ & $74,00 \mathrm{a}$ & $60,00 \mathrm{a}$ \\
Transversal & 10,80 & 23,00 & 104,00 & 80,00 \\
{$\left[\alpha_{\mathrm{r}}\right]$} & & & & \\
\hline Fonte: Adaptado de ACI Committee $440[28]$. &
\end{tabular}

\subsection{Comportamento à tração}

De forma geral, em comparação a barras de aço convencionalmente utilizadas como armaduras no concreto armado, barras de GFRP apresentam maior resistência a tração e menor módulo de elasticidade. A Tabela 04 as propriedades mecânicas de tração para as barras GFRP, aço e demais fibras [28].

Quando submetidos a um carregamento, as barras GFRP não contemplam comportamento plástico (escoamento) antes da ruptura. Tal comportamento à tração das barras GFRP é caracterizado por uma relação elástica linear de tensãodeformação até a falha. A figura 5 apresenta o diagrama de tensões e deformações específicas para o aço e barra FRP onde pode-se observar o comportamento das barras GFRP perante os demais. Cabe ressaltar que enquanto o aço apresenta um comportamento elasto-plástico (dúctil), as barras de GFRP e demais apresentam um comportamento elasto-frágil [28]. 


\section{REVISTA}
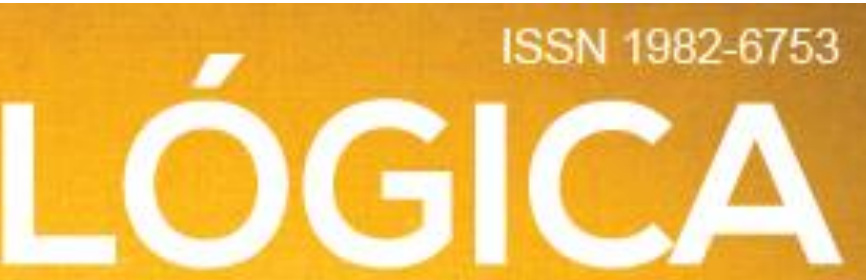

Tabela 4: Coeficientes de expansão térmica nas direções transversal e longitudinal.

\begin{tabular}{|c|c|c|c|c|}
\hline & Aço & GFRP & CFRP & AFRP \\
\hline Tensão de escoamento [Mpa] & 276 a 517 & N/A & N/A & N/A \\
\hline Resistência à tração [Mpa] & 483 a 690 & 483 a 1600 & 600 a 3690 & 1720 a 2540 \\
\hline Módulo de elasticidade [GPa] & 200 & 35 a 51 & 120 a 580 & 41 a 125 \\
\hline Deformação no escoamento [\%] & 0,14 a 0,25 & N/A & N/A & N/A \\
\hline Deformação na ruptura [\%] & 6,0 a 12,0 & 1,2 a 3,1 & 0,5 a 1,7 & 1,9 a 4,4 \\
\hline
\end{tabular}

* Valores típicos para uma relação volumétrica das fibras variando de 0,5 a 0,7.

** N/A: Não apresenta.

Fonte: Adaptado de ACI Committee 440 [28].

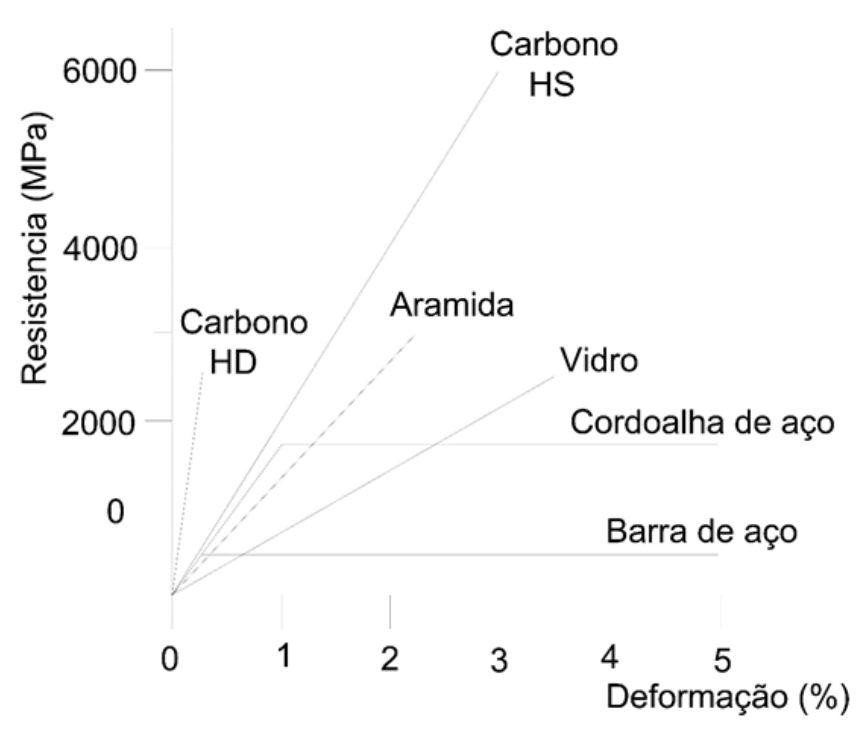

Figura 5: Diagrama tensão x deformação. Fonte: Adaptado de ACI Committee 440 [28].

A resistência a tração e rigidez de uma pode ser afetada por alguns fatores. Como a resina possui uma resistência menor que as fibras, a fração volumétrica de fibras par com o volume geral afeta significativamente as propriedades de tração da barra. A taxa de cura, o controle de qualidade e processo de fabricação acabam por também afetar as características mecânica das barras [30]. Cabe ressaltar que a resistência a tração de barra GFRP se dá em função do seu diâmetro da barra, logo barras esguias correspondem a maior resistência, fato que pode ser observado na Tabela 5 .

Ao menos que tenha uma resina termoplástica em sua composição, as barras de GFRP não devem serem dobradas pós fabricação. Todavia, há exceções que podem ser fabricadas com dobras, sendo que a mesma pode ter uma redução de $40 \%$ a $50 \%$ da resistência a tração em comparação a barra reta devido a concentração de flexão e tensão da fibra [31].

Tabela 4: Resistência à tração mínima

\begin{tabular}{ccc}
\hline \multirow{2}{*}{ Conversão métrica } & Diâmetro nominal & $\begin{array}{c}\text { Resistência à tração } \\
\text { mínima }\end{array}$ \\
\cline { 2 - 3 } & {$[\mathrm{mm}]$} & GFRP [Mpa] \\
\hline $\mathrm{N}^{\circ} .6$ & 6,40 & 760 \\
$\mathrm{~N}^{\circ} .10$ & 9,50 & 760 \\
$\mathrm{~N}^{\circ} .13$ & 12,70 & 690 \\
$\mathrm{~N}^{\circ} .16$ & 15,90 & 655 \\
$\mathrm{~N}^{\circ} .19$ & 19,10 & 620 \\
$\mathrm{~N}^{\circ} .22$ & 22,50 & 586 \\
$\mathrm{~N}^{\circ} .25$ & 25,40 & 550 \\
$\mathrm{~N}^{\circ} .29$ & 28,70 & 517 \\
$\mathrm{~N}^{\circ} .32$ & 32,30 & 480
\end{tabular}

Fonte: Adaptado de ACI Committee 440, 2015.

\subsection{Comportamento à compressão}

Em quesito de resistência a compressão, as barras GFRP apresentam um comportamento inferior à sua resistência a tração. Experimentalmente, Mallick [32] e Wu [30] constataram que a resistência a compressão é de aproximadamente $55 \%, 78 \%$ e $20 \%$ da resistência a tração para GFRP. CFRP e AFRP respectivamente. Destarte, não aconselha a utilização de barras GFRP e similares para resistir a compressão. 


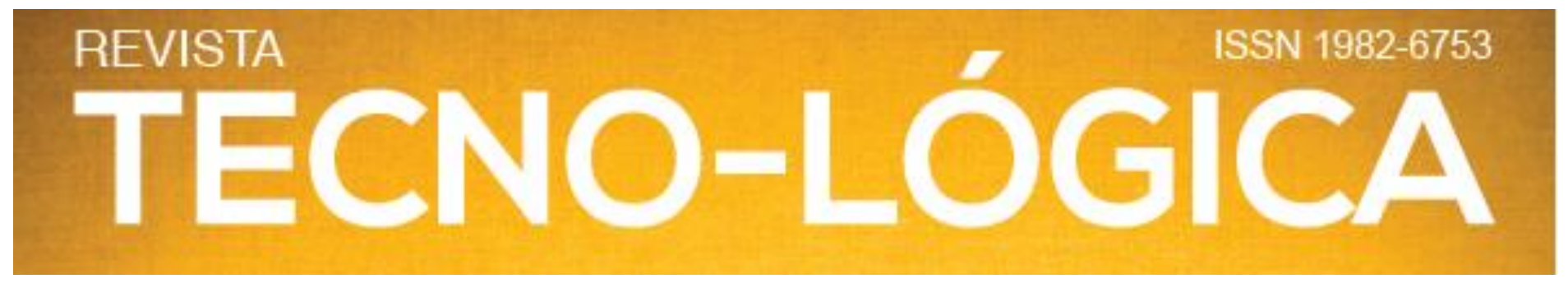

Quando submetidas a compressão longitudinal pode-se apresentar falhas transversal a tração, microflambagem da fibra ou falha por cisalhamento. Logo, o modelo de falha é consoante ao tipo de fibra, a fração volumétrica das fibras e tipo de resina.

Consequentemente, tais barras apresentam um módulo de elasticidade na compressão de menor magnitude em relação ao módulo de elasticidade a tração. Estudo desenvolvidos por Mallick [32] e Ehsani [33] mostraram que o módulo de elasticidade a compressão é aproximadamente $80 \%$ do módulo de elasticidade a tração para barras GFRP.

\subsection{Comportamento ao cisalhamento}

A barras GFRP são relativamente fracas ao cisalhamento interlaminar, onde os espaços entre as fibras se encontram preenchidos por camada de resina não reforçada. Devido ao fato de, geralmente, não haver tal reforço nessas regiões, a resistência ao cisalhamento é regida pela matriz polimérica a qual apresentam pouca resistência [28]. Através de tranças ou enrolamento de fibras transversais ao núcleo de fibras principais, a depender do grau de deslocamento, pode-se aumentar a resistência ao cisalhamento devido a orientação das fibras, inseridas fora do eixo, estarem dispostas entre as fibras principais [28]. Tal processo também pode ser feito por meio da introdução de um tapete de fios contínuos junto ao processo de pultrusão.

\subsection{Aderência}

A aderência de uma barra GFRP junto ao concreto depende do processo de fabricação empregado, das propriedades mecânicas das barras, condições ambientais, bem como, das caraterísticas da superfície. A força de aderência oriunda da ancoragem das barras GFRP junto ao concreto pode ser transmitida pela resistência de adesão da interface, denominada também como adesão química, resistência da interface ao atrito ou intertravamento mecânico [28].

Ressalta-se que, tais barras podem apresentar uma falha de aderência na resina, porventura da resistência a aderência ser transmitida da resina para a fibra.

\section{Comportamento ao longo do tempo}

A deterioração ao longo do tempo é algo congênito em estruturas, todavia, estruturas em concreto armado com barras de GFRP apresentam deterioração em menor escala que em estruturas de concreto convencionais.

\subsection{Fadiga}

De forma geral, o comportamento a fadiga das fibras de vidro, porventura de sua suscetibilidade a umidade e soluções ácidas e alcalinas, a determinação de um limite a fadiga torna-se difícil de ser determinado.
Em um milhão de ciclos de carregamentos, a resistência a fadiga encontra-se na faixa de $60 \%$ a $70 \%$ da resistência máxima estática inicial [28]. Para que a resistência a fadiga seja afetada a interface resina ou resina/fibra dever-se-á estar degrada pelo ambiente, do contrário a mesma permanece imutável.

As fibras de vidro individuais em si, baseado em experimentos laboratoriais, apresentam uma ruptura retardada devido a corrosão por tensão, para quantidades mínimas de umidade a quais foram expostas porventura de falhas na superfície. Ressalta-se que a incorporação de fibras de vidro junto a barras, para quesito de efeito cíclico de fadiga à tração, observara-se perdas na resistência estática inicial de até $10 \%$ por década sw vida útil logarítmica [34]. Atribui-se tal efeito a interação fibra-fibra sem estar atrelado a corrosão por tensão descrito para fibras individuais.

\subsection{Durabilidade}

Ao ser exposto a ações intempéricas como temperaturas elevadas, soluções químicas e umidade, as propriedades mecânicas de muitos sistemas GFRP acabam sendo reduzidas. Tal redução é consoante a zona de imersão, duração da exposição, tipo de fibra constituinte, formulação da resina e o método de cura da resina.

Logo, a resistência a tração e rigidez das barras podem ser degradadas porventura da exposição prolongada junto a soluções aquosas básicas de $\mathrm{PH}$ elevado e altas temperaturas. Tal fato fora comprovado através de estudos como de Porter e Barnes (43) que obtiveram reduções de $0 \%$ a $75 \%$ da resistência a tração inicial e reduções de $0 \%$ a $20 \%$ da rigidez elástica inicial utilizando com objeto de estudo barras com fibra de vidro (VPRF). Já com barras com fibra de aramida (APRF), Takewaka e Khin [35], Rostasy [36] e Sen et at [38] demostraram reduções de $10 \%$ a $50 \%$ para tração e de $0 \%$ a $20 \%$ para rigidez elástica em contrapartida aos valores iniciais. Ainda em estudos de Takewaka e Khin [35], todavia para barras com fibra de carbono (CPRF) fora obtido reduções para tração e rigidez de $0 \%$ a $20 \%$ em relação aos valores iniciais.

Por conseguinte, a exposição do material a raios ultravioletas e umidade antes ainda de sua disposição final junto ao concreto, acaba por comprometer as propriedades dos componentes do polímero. Em experimentos de exposição a tais agentes, Sasaki et al [39] e Uomoto [40], observaram reduções para VPRF, APRF e CPRF de $40 \%, 35 \%$ e $20 \%$ respectivamente em comparação a resistência inicial.

A efetividade da aderência das barras GFRP junto ao concreto depende da transferência dos esforços cortantes entre a barra, concreto e entre as fibras constituintes da barra. Ambientes úmidos e alcalinos os quais degradam a resina do GFRP ou a interface da fibra/resina, por conseguinte, acabam degradando a 


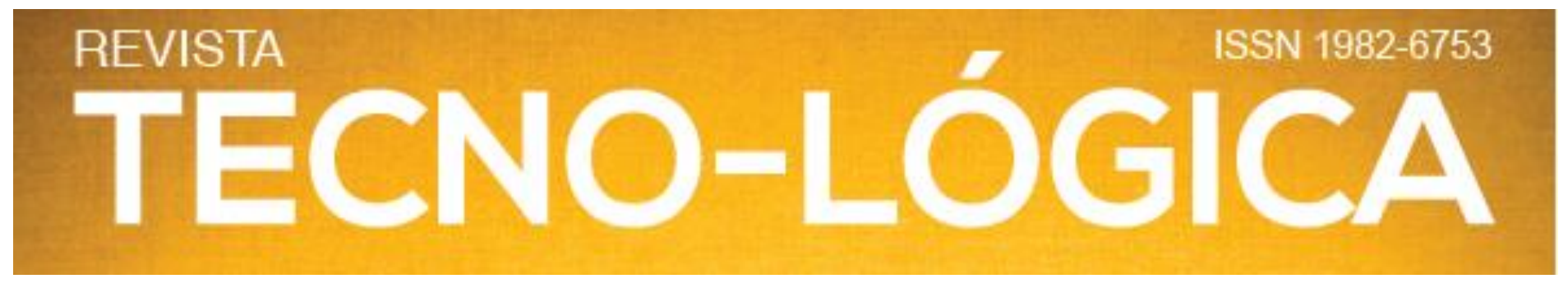

aderência da barra. Testes de arrancamento realizado em barras CPRF e VPRF sob condições ambientais não indicaram reduções expressivas para quesito de aderência em um intervalo de 1 a 2 anos [37, 41].

\section{Conclusões}

Nas últimas décadas o interesse por materiais resilientes e de alto desempenho aumentou significativamente. Pois, cada vez mais, as obras de construção civil apresentam uma elevada complexidade de execução, ora por condições climáticas adversas, ora por questões econômicas e por durabilidade.

A durabilidade do concreto armado está associada intimamente com a corrosão da armadura do aço, ocasionada por agentes deletérios. Perante esta perspectiva, as barras de GFRP se apresentam como uma alternativa promissora em substituição da armadura de aço. Outras vantagens que o material apresenta é de não ser magnético e muito menos condutor elétrico, isso o torna relevante em muitas outras áreas do conhecimento, como medicina nuclear, química e entre outros.

Entretanto, o plano de fundo deste estudo é prover um estudo referencial aprofundado das barras GFRP, visando fomentar novos estudos sobre a temática. Mesmo sendo vantajoso, quando falamos desta técnica construtiva é importante priorizar que a composição das barras deriva trivialmente de dois materiais relativamente frágeis. E atualmente, existe uma carência de estudos aprofundados de CA GFRP.

Este artigo, vislumbra ser um ponto inicial no desenvolvimento de uma norma brasileira de dimensionamento de CA GFRP. Sendo ele, uma parte inicial da solução de inúmeros problemas patológicos e de falta de resiliência existente em boa parte das obras e sistemas de infraestrutura brasileira. Visando que no futuro o gerenciamento dos ativos de infraestrutura, sejam mais viáveis economicamente, sem comprometer a segurança e qualidade dos serviços, e sem onerar os cofres públicos.

\section{FIBERGLASS BARS, AN INNOVATIVE ALTERNATIVE AND ITS POTENTIAL: LITERATURE REVIEW}

ABSTRACT: The performance, efficiency, resilience and sustainability of a structure are fundamental parameters to promote the quality of life and economic development of an infrastructure. Currently, pathologies such as corrosion are serious problems that burden public coffers and the final consumer, in addition to generating greater annoyances over time. Therefore, the use of alternative and high-performance materials is gaining more and more attention, due to the high complexity of management, maintenance and resilient adaptation to social and climatic changes, accentuated pollution, decreased financial resources and among others throughout its cycle infrastructure life. Therefore, GFRP (Glass-Fiber-Reinforced Polymer) bars gain evidence for being corrosive inert, being used as a tool for repair, maintenance and employment in new civil works. This technology-associated material enables cost reduction and improvement in the structure's behavior. The background of this article is to encourage future studies, aiming to develop a Brazilian standard for sizing reinforced concrete (CA) GFRP. The mastery of this subject to professionals, managers and those involved in the design and implementation of infrastructure systems and civil works is essential, as it allows for systemic planning, optimized and consistent with needs, minimizing costs, but without compromising the quality of the structure.

Keywords: GFRP reinforcement bars. Fiber reinforced concrete. Glass fiber reinforced polymer GFRP.

\section{Referências}

[1] KOCAOZ, S., SAMARANAYAKE, V. A., NANNI, A., "Tensile characterization of glass FRP bars", Composites: Part B engineering, v. 36, pp. 127 - 134, Março 2005

[2] RIBEIRO, S. E. C. “Análise da confiabilidade de vigas de concreto armado com plástico reforçado por fibras". Tese de D. Sc., Universidade Federal de Minas Gerais, Belo Horizonte, MG, Brasil, 2009.

[3] SOLYOM, S., DI BENEDETTI, M., GUADAGNINI, M., et al., "Effects of temperature on the concrete GFRP bar union behavior", Composites Part B, v. 183, pp. 107602, Fevereiro 2020

[4] JIA, D., GUO, Q., MAO, J., et al., "Durability of glass fibre-reinforced polymer (GFRP) bars embedded in concrete under various environments. I: Experiments and analysis", Composite Structures, v. 234, pp. 111687, 2020.

[5] RIBEIRO, B. C., LOPES, R. M., RICCIO, V. A., et al., “Análise teórica de vigas de concreto armadas com barras de plástico reforçado com fibras”, Revista Construindo, v. 8, n. 02, Julho/Dezembro 2016.

[6] BRITISH STANDARTS ASSOCIATION, "Structural Use of Concrete", BS8110, London, 1997.

[7] EGYPTIAN MINISTRY OF HOUSING, UTILITIES, AND URBAN DEVELOPMENT, "Egyptian Code for Design and Construction of Concrete Structures". Giza, Egito, 2001.

[8] EGYPTIAN MINISTRY OF HOUSING, UTILITIES, AND URBAN DEVELOPMENT. Egyptian Code for the Use of Fiber Reinforced Polymers (FRP) in the Construction Fields, Egyptian Housing and Building National Research Center, Giza, Egito, 2005.

[9] SÁ, M. F., GOMES, A. M., CORREIA, J. R., et al., "Creep behavior of pultruded GFRP elements - Part 1: Literature review and experimental study", Composite Structures, v. 93, n. 10, pp. 2450-2459, Setembro 2011.

[10] CORREIA, M. M., "Comportamento estrutural de perfis pultrudidos de GFRP: Caracterização experimental e modelação numérica”, Dissertação de M.Sc., Universidade Técnica de Lisboa, Lisboa, Portugal, 2012.

[11] NAYAK, R. K. "Influence of seawater aging on mechanical properties of 


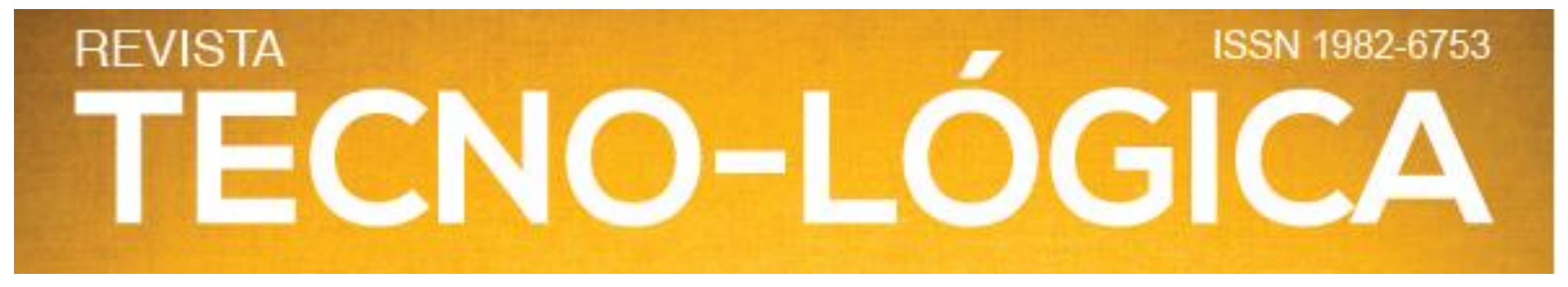

nano-A12O3 embedded glass fiber reinforced polymer nanocomposites", Construction and Building Materials, v. 221, n. 10, pp. 12-19, Outubro, 2019.

[12] ROSA, I. C. M. "Comportamento mecânico de materiais compósitos FRP a temperatura elevada". Dissertação de M. Sc., Universidade de Lisboa, Lisboa, Portugal, 2016.

[13] COUTO, I. A., “Análise teórica e experimental do comportamento da aderência entre o concreto e barras de fibra de vidro impregnada por polímero", Dissertação de M.Sc., Universidade de São Paulo, São Carlos, SP, Brasil, 2007.

[14] SÁ, M. F., SILVESTRE, N., CORREIA, J. R., et al., "Dynamic behaviour of a GFRP-steel hybrid pedestrian bridge in serviceability conditions. Part 2:

Numerical and analytical study". Thin-Walled Structures, v. 118, pp. 113-123, Setembro 2017.

[15] BAZLI, M., ZHAO, X. L., BAI, Y., et al., "Durability of pultruded GFRP tubes subjected to seawater sea sand concrete and seawater environments", Construction and Building Materials, v. 245, pp. 118399, Junho 2020.

[16] BENMOKRANE, B., WANG, P., TON-THAT, T. M., et al., "Durability of glass fiber-reinforced polymer reinforcing bars in concrete environment", Journal of Composites for Construction, v. 6, n. 3, pp. 143-153, Agosto 2002.

[17] CANADIAN STANDARTS ASSOCIATION (CSA S806), "Canadian highways bridge design code", section 16- Fibre reinforced structures. Final draft, 1996.

[18] FEDERATION INTERNATIONALE DU BETON. "FIB 9.3 TG - FRP as reinforcement in concrete", (Proposta de norma), Sheffield, Inglaterra, 2003.

[19] MACHADO, A. P. "Reforço de Estruturas de Concreto Armado com Fibras de Carbono", São Paulo, Pini, 2002.

[20] TIGHIOUART, B., BENMOKRANE, B., GRAO, D., "Investigation of bond in concrete with fiber reinforced polymer (FRP) bar", Construction Building and Materials, v. 12, n. 8, pp. 453-462, Dezembro 1998.

[21] BAKIS, C. E., BANK, L. C., ASCE, F., et al., "Fiber reinforced polymer composites for construction - State-of-the-art review", Journal of composites for Construction, v. 6, n. 2, pp. 73-87, Maio 2002.

[22] BANK, L. C., "Properties of FRP Reinforcement for Concrete," FiberReinforced-Plastic (FRP) Reinforcement for Concrete Structures: Properties and Applications, Developments in Civil Engineering, V. 42, A. Nanni, ed., Elsevier, Amsterdam, pp. 59-86, Maio 1993.

[23] MASMOUDI, R.; ZAIDI, A.; GÉRARD, P.. "Transverse Thermal Expansion of FRP Bars Embedded in Concrete". Journal Of Composites For Construction, [s.1.], v. 9, n. 5, p. 377-387, out. 2005. American Society of Civil Engineers (ASCE). http://dx.doi.org/10.1061/(asce)1090-0268(2005)9:5(377).

[24] GENTRY, T. Russell; HUSAIN, "Mohamed. Thermal Compatibility of Concrete and Composite Reinforcements". Journal Of Composites For Construction, [s.1.], v. 3, n. 2, p. 82-86, maio 1999. American Society of Civil Engineers (ASCE). http://dx.doi.org/10.1061/(asce)1090-0268(1999)3:2(82).

[25] BELLAKEHAL, H.; ZAIDI, A.; MASMOUDI, R.; BOUHICHA, M.. "Combined effect of sustained load and freeze-thaw cycles on one-way concrete slabs reinforced with glass fibre - reinforced polymer". Canadian Journal Of Civil Engineering, [s.1.], v. 40, n. 11, p. 1060-1067, nov. 2013. Canadian Science Publishing. http://dx.doi.org/10.1139/cjce-2012-0514.

[26] ZAIDI, Ali; MASMOUDI, Radhouane; BOUHICHA, Mohamed. Numerical analysis of thermal stress-deformation in concrete surrounding FRP bars in hot region. Construction And Building Materials, [s.1.], v. 38, p. 204-213, jan. 2013. Elsevier BV. http://dx.doi.org/10.1016/j.conbuildmat.2012.08.047.

[27] ZAIDI, A.; MASMOUDI, R.. Thermal effect on fiber reinforced polymer reinforced concrete slabs. Canadian Journal Of Civil Engineering, [s.1.], v. 35, n. 3, p. 312-320, mar. 2008. Canadian Science Publishing. http://dx.doi.org/10.1139/107-110.

[28] AMERICAN CONCRETE INSTITUTE. ACI 440.1R-15: Guide for the Design and Construction of Structural Concrete Reinforced with FiberReinforced Polymer (FRP) Bars. Detroit (USA): ACI, 2015. 88 p.

[30] Wu, W. P., "Thermomechanical Properties of Fiber Reinforced Plastic (FRP) Bars," PhD dissertation, West Virginia University, Morgantown, WV, 292 p, 1990.

[31] NANNI, A.; RIZKALLA, S.; BAKIS, C. E.; CONRAD, J. O.; ABDELRAHMAN, A. A., "Characterization of GFRP Ribbed Rod Used for Reinforced Concrete Construction," Proceedings of the International Composites Exhibition (ICE-98), Nashville, TN, pp. 16A/1-6, 1998.

[32] MALLICK, P. K., Fiber Reinforced Composites, Materials, Manufacturing, and Design, Marcell Dekker, Inc., New York, 469 p, 1988.

[33] EHSANI, M. R., "Glass-Fiber Reinforcing Bars," Alternative Materials for the Reinforcement and Prestressing of Concrete, J. L. Clarke, Blackie Academic \& Professional, London, UK, pp. 35-54,1993.

[34] MANDELL, J. F., "Fatigue Behavior of Fiber-Resin Composites," Developments in Reinforced Plastics, V. 2, Applied Science Publishers, London, p. 67-107, 1982.

[35] TAKEWAKA, K., AND KHIN, M., "Deterioration of Stress-Rupture of FRP Rods in Alkaline Solution Simulating as Concrete Environment," Advanced Composite Materials in Bridges and Structures, M. M. El-Badry, ed., Canadian Society for Civil Engineering, Montreal, QC, Canada, p. 649-664, 1996.

[36] ROSTASY, F. S.,"On Durability of FRP in Aggressive Environments," Proceedings of the Third International Symposium on Non-Metallic (FRP) Reinforcement for Concrete Structures (FRPRCS-3), V. 2, Japan Concrete Institute, Tokyo, Japan, p. 107-114, 1997.

[37] SEN, R.; SHAHAWY, M.; SUKUMAR, S.; ROSAS, J., "Effect of Tidal Exposure on Bond of CFRP Rods," Second International Conference on Composites in Infrastructure, V. II, H. Saadatmanesh and M. R. Eshani, eds., University of Arizona, Tucson, AZ, p. 512-523, 1998a.

[38] SEN, R.; SHAHAWY, M.; ROSAS, J.;SUKUMAR, S.,"Durability of Aramid Pretensioned Elements in a Marine Environment," ACI Structural Journal, V. 95, No. 5, Sept.-Oct., p. 578-587, 1998 b.

[39] SASAKI, I.; NISHIZAKI, I.; SAKAMOTO, H.; KATAWAKI, K.;KAWAMOTO, Y., "Durability Evaluation of FRP Cables by Exposure Tests," Proceedings of the Third International Symposium on Non-Metallic (FRP) Reinforcement for Concrete Structures (FRPRCS-3), V. 2, Japan Concrete Institute, Tokyo, Japan, pp. 131-137, 1997.

[40] UOMOTO, T., "Durability of FRP as Reinforcement for Concrete Structures," Proceedings of the 3rd International Conference on Advanced Composite Materials in Bridges and Structures, ACMBS-3, J. L. Humar and A. G. Razaqpur, eds., Canadian Society for Civil Engineering, Montreal, QC, Canada, pp. 3-17, 2000.

[41] CLARKE, J., SHEARD, P., "Designing Durable FRP Reinforced Concrete Structures," Proceedings of the First International Conference on Durability of Composites for Construction, B. Benmokrane and H. Rahman, eds., Sherbrooke, QC, Canada, p. 13-24, 1998.

[42] ISTRUDTE, Interim Guidance on the Design of Reinforced Concrete Structures using Fibre Composite Reinforcement, Institution of Structural Engineers, London, 116 pp, 1999.

[43] PORTER, M. I., \& BARNES, B. A, Accelerated Durability of FRP Reinforcement for concrete Structures. Proceedings of the First International Conference on Composites for Construction (CDCC 1998). Sherbrooke, Québec, pp 191-198. 